\title{
Status of LIGO
}

\section{Gary H. Sanders}

Gary H. Sanders, "Status of LIGO," Proc. SPIE 4856, Gravitational-Wave Detection, (26 February 2003); doi: 10.1117/12.459097

Event: Astronomical Telescopes and Instrumentation, 2002, Waikoloa, SPIE. Hawai'i, United States 


\title{
Status of LIGO
}

\author{
Gary H. Sanders* \\ Laser Interferometer Gravitational Wave Observatory (LIGO), California Institute of Technology
}

\begin{abstract}
LIGO construction has been completed. The three interferometers at the two LIGO observatory sites (Livingston, Louisiana and Hanford, Washington) have been operated successfully as power-recycled Michelson interferometers with Fabry-Perot arm cavities. Commissioning of the interferometers has progressed to operating them simultaneously in this final optical configuration. The initial coincidence operation between the observatory sites has provided a full test of the detector hardware and software subsystems, and full operation of the data acquisition and data analysis systems. The LIGO Laboratory and the LIGO Scientific Collaboration are working together to exploit the early series of interleaved engineering and science runs to commission the detector and data systems, to provide a detailed characterization of the detector and to produce the first scientific results from LIGO. The operation of LIGO is also coordinated with operation of the GEO 600 detector and the ALLEGRO resonant mass detector. The status of this early operation and data study will be presented.
\end{abstract}

Keywords: gravity, general relativity, gravitational waves, LIGO, Virgo, GEO, TAMA

\section{LIGO OVERVIEW}

Massive cosmic objects undergoing violent relativistic motions emit gravitational waves. These waves may be emitted by a variety of astrophysical sources. For example, they have been shown to account for the emission of energy from relativistic neutron star binary systems ${ }^{1}$. In reaching that result, gravitational wave emissions were shown to provide the dissipative mechanism that leads to the ultimate inspiral and collapse of these systems. This result was accomplished by precision measurements of the long-term evolution of the orbital period of a binary system, revealing the decay of the orbit. However, near the endstage of the inspiral the system becomes ultrarelativistic and the resulting extremely strong gravitational waves emitted in the final moments before collapse are predicted to be strong enough for direct detection on Earth ${ }^{2}$. Terrestrial detectors must be able to detect these time-varying disturbances of space-time. These waveforms contain information about the dynamical behavior of the emitting source. For the example given, the waves are expected to lie in the audio frequency range.

The Laser Interferometer Gravitational Wave Observatory (LIGO) ${ }^{3}$ consists of two observatories that support groundbased gravitational wave detectors sensitive in the audio frequencies. These facilities (stable platforms and ultrahigh vacuum environments) provide stable vibrationally quiet platforms adding little compromise to the local environmental disturbances that ultimately limit the sensitivity of the most advanced ground-based detector. The observatories are sited approximately $3000 \mathrm{~km}$ apart in order to permit the formation of a long-baseline coincidence between candidate gravitational wave signals from individual detectors. This enables rejection of local disturbances that satisfy the event selection process for individual site data. Figure 1 displays the location of the two LIGO observatories, near Hanford, Washington and Livingston, Louisiana.

The observatory facilities are able to support the current initial set of detectors, and future detectors that may ultimately achieve the sensitivity possible at these locations. This paper discusses the status of the initial set of LIGO interferometric detectors. However, an active program of research and development is underway that will underpin the second generation of LIGO detectors ${ }^{4}$. These will be installed following the full initial LIGO science run.

\footnotetext{
* sanders@ligo.caltech.edu; phone 1-626-395-2997; fax 1-626-304-9834; http://www.ligo.caltech.edu; LIGO Laboratory, California Institute of Technology, MC 18-34, Pasadena, CA 91125 USA
} 


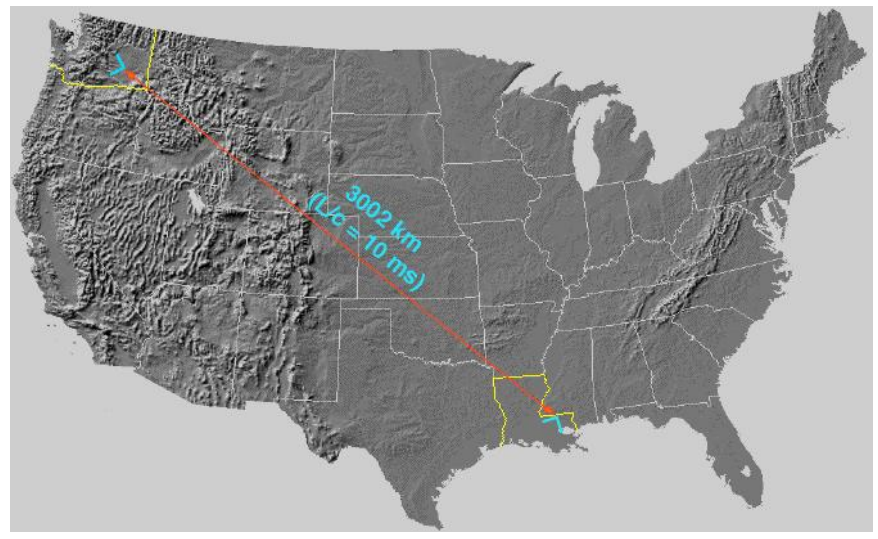

Figure 1 The two LIGO observatories provide a $3000 \mathrm{~km}$ baseline for eliminating background signals detected only at a single observatory.

The initial set of LIGO detectors consists of three Michelson interferometers with arms comprised of Fabry-Perot cavities. Two interferometers, with $4 \mathrm{~km}$ and $2 \mathrm{~km}$ cavity lengths respectively, are located in the vacuum system at the Hanford observatory. A single $4 \mathrm{~km}$ arm cavity instrument is located at Livingston. The two instruments at Hanford provide some additional ability to reject background signals.

Figure 2 displays a schematic layout of an initial LIGO interferometer. Passing gravitational waves appear as timevarying departures from the flat space-time metric, described as the dimensionless quantity $\operatorname{strain}^{2}$. The waves cause differential changes in the orthogonal arm cavity lengths. The Fabry-Perot optical cavities in the arms allow a buildup of the optical power. Storage time of the light in the arm cavities is sufficient to sample the full phase change in the recombined differential arm signals. With sufficient phase sensitivity and displacement sensitivity, such an interferometer can sense the strain signal.

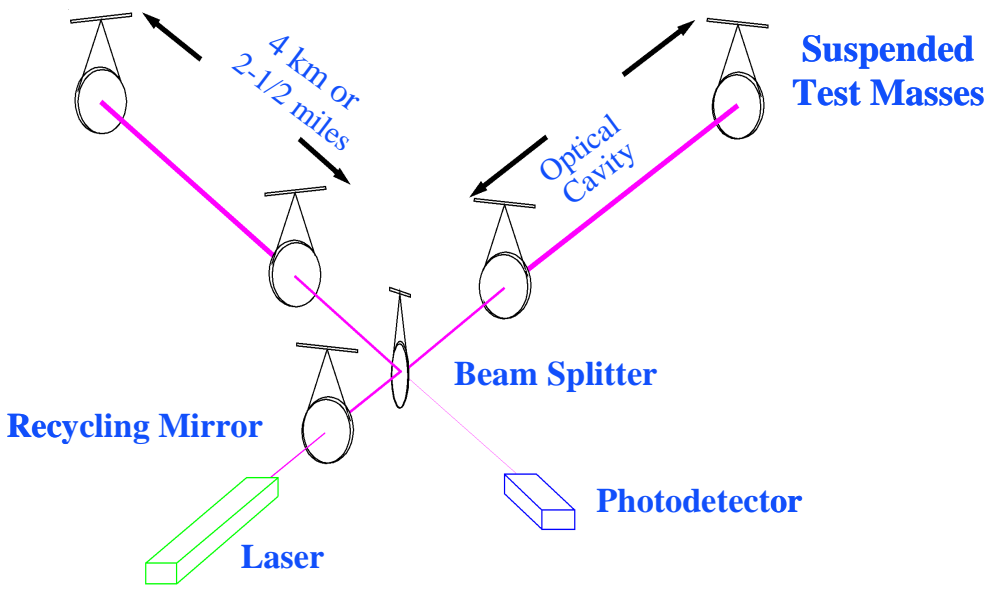

Figure 2 Schematic layout of a LIGO interferometer consisting of a Michelson interferometer with Fabry-Perot arm cavities. The addition of a mirror at the entrance to the interferometer serves to recycle light reflected back to the source laser.

An additional feature of the LIGO interferometers is the use of a recycling mirror to return light back to the interferometer that is exiting the beam splitter and returning to the laser, effectively multiplying the laser power by the recycling cavity gain. 
Figure 3 displays the spectrum of strain sensitivity of the initial LIGO interferometers as a function of the gravitational wave frequency ${ }^{5}$ as limited by the main sources of noise in the interferometer and facility. The noise budgets for individual contributions to the noise floor are shown.

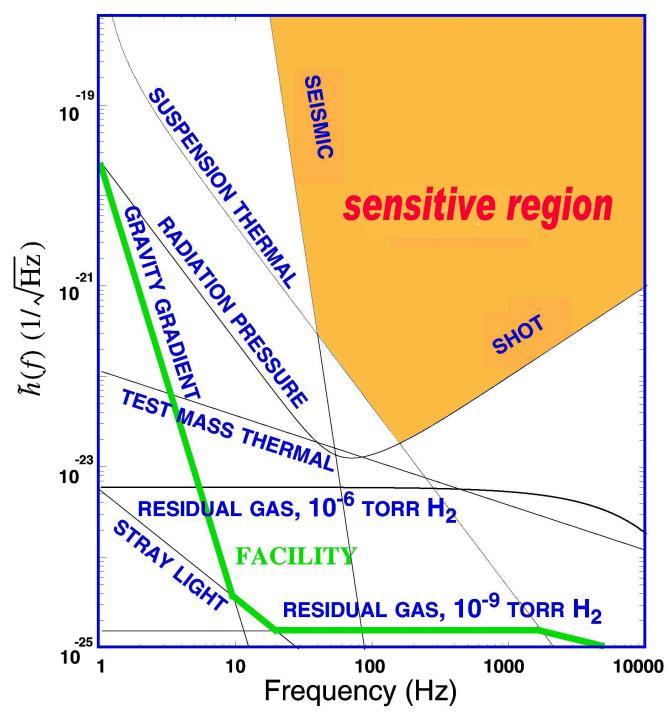

Figure 3 Initial LIGO interferometer spectrum of strain noise and the separate contributions from the principal noise sources. At the lowest frequencies, initial LIGO interferometers are limited by seismic disturbances transmitted through isolation system. At the highest frequencies, the photon counting statistics, shot noise, limits the precision of the measurement. In the middle frequencies, thermally driven mechanical excitations in the test mass mirror suspensions determine the noise floor. Below these limiting mechanisms, contributors to the noise derive from quantum mechanical radiation pressure fluctuations, and thermally driven excitations of the mirror reflecting surfaces. The latter two mechanisms are related to the interferometer design. The facility contributes to the noise floor as well. Index of refraction fluctuations from the residual gas in the beam tube path sets a noise floor shown for two levels of vacuum pressure. Stray light scattered from the interferometer optical path can also reenter the measurement. Finally, classical gravity gradients from surrounding terrestrial mass set a limit that is the property of any earthbound detector.

With the initial LIGO design sensitivity, the predicted event rates for various gravitational wave sources has been estimated by many authors ${ }^{6}$. Initial LIGO has a plausible chance to detect the predicted sources. Future upgrades will provide a likely observation ${ }^{4}$.

\section{LIGO CONSTRUCTION}

LIGO construction is essentially complete. Significant construction activity began in late 1994 but this construction followed an extensive R\&D phase reaching back to the first ideas for a LIGO system ${ }^{7}$.

Constructing LIGO consisted of two parallel subprojects. One subproject provided the facilities (technical foundations, arm slabs, buildings, vacuum system, beam tubes, beam tube enclosures) that can house the successive generations of detectors. The other subproject completed the remaining preconstruction $R \& D$ for the initial interferometers, and fabricated the interferometer components ready for installation at the two observatories. These two subprojects were managed as a single project using conventional industrial techniques for controlling the technical, cost and schedule progress of the fully planned and baselined project. Figure 4 displays the cost and schedule progress of the LIGO construction project and provides a comparison of these with the planned and controlled project baseline. The fabricated 
interferometer components have been installed into the facilities as the first activities of the LIGO early operations and commissioning phase.

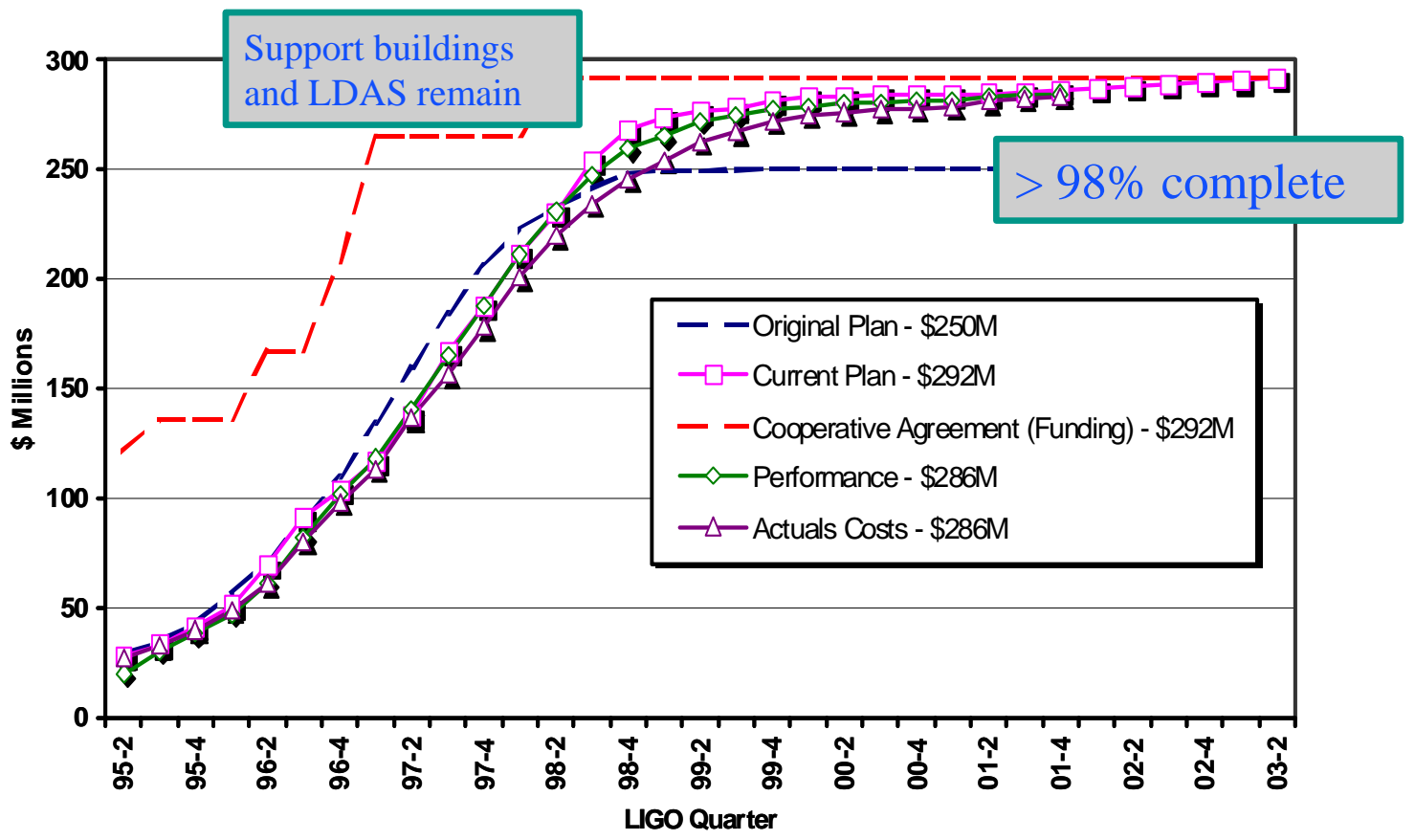

Figure 4 Performance measurement plot of the LIGO construction project. The funding provided under the US National Science Foundation Cooperative Agreement is shown as a function of time, reaching the ceiling budget of \$292 million. The Original Plan baseline represents the time-phased budget for all construction project work as planned in 1995 and placed under project baseline control. The difference between the integrated Original Plan budget and the NSF funding is held in a contingency fund and allocated to manage risk during project execution. The Current Plan plot represents the revised time-phased budget after successive contingency allocation actions during the project. The Performance curve represents the earned value of work completed in the project as a function of time. The Actuals Costs curve represents the paid costs for this completed and earned value. At this time, completion of the last laboratory support buildings and the final purchases for the LIGO Data Analysis System (LDAS) are the main remaining items to complete. The project is more than $98 \%$ complete at this stage.

Early operations have involved installing the interferometer components, separately commissioning the subsystems, and, in turn, commissioning each of the three interferometers. The interferometer installation and commissioning commenced with the Hanford $2 \mathrm{~km}$ interferometer, followed by the Livingston $4 \mathrm{~km}$ instrument. Finally, employing many lessons learned from the earlier interferometers and employing a new generation of digital suspension control electronics, the Hanford $4 \mathrm{~km}$ interferometer was brought into operation.

It should be noted that among large scientific facility projects, it is rare that several major systems are commissioned in overlapping periods by the same project team. This presents a challenge in managing the rapid completion of components for all systems, and avoiding the installation of components whose design could be informed by the early commissioning experience. Setting the right balance between early parallel completion and phased completion that incorporates lessons learned in the first phases paces the LIGO installation and commissioning and challenges the expert technical teams. 
Table 1 lists, in summary form, the main construction, installation and commissioning activities by year for the project, and the envisioned period of scientific operations with the initial detector systems. In greater detail, each of the main activities overlapped in time, but the table displays the main project focus for the listed years.

Table 1 Main activities of LIGO construction and operations for the listed years. During construction, the LIGO project consisted of two subprojects to deliver the facilities and the initial set of LIGO detector systems.

\begin{tabular}{|c|l|l|}
\hline Year & \multicolumn{1}{|c|}{ Facility Subproject Major Activities } & \multicolumn{1}{|c|}{ Detector Subproject Major Activities } \\
\hline & & \\
\hline 1996 & Civil works, foundations, slabs & Preconstruction R\&D and design \\
\hline 1997 & Building construction & Preconstruction R\&D and design \\
\hline 1998 & Vacuum and beam tube systems & Fabrication \\
\hline 1999 & Vacuum and beam tube systems & Initiate installation into vacuum system \\
\hline 2000 & Facility construction complete & Commissioning subsystems, first lock achieved \\
\hline 2001 & & First coincidence engineering run \\
\hline 2002 & & Sensitivity studies, initiate science operations \\
\hline 2003 & & Sensitivity studies, science operations \\
\hline $2004-2006$ & & Science operations \\
\hline
\end{tabular}

\section{STATUS OF COMMISSIONING LIGO}

The first operation of an entire LIGO interferometer took place in October 2000 when the Hanford $2 \mathrm{~km}$ interferometer achieved "first lock". The central Michelson cavity, and both Fabry-Perot arm cavities were simultaneously placed in resonance. This recombined configuration was later operated with the power-recycling cavity formed with the recycling mirror to achieve power-recycled operation. Since that milestone, commissioning of the $2 \mathrm{~km}$ interferometer has been extended to long durations, with progressive additions of additional servo loops and improvements in several subsystems. The goal of this commissioning effort has been to lower the interferometer noise floor, achieve lock acquisition more readily, and to extend the uninterrupted periods of stable, sensitive locked operation. Furthermore, this first interferometer commissioning guides many of the systems and techniques employed in the subsequent efforts at the other two LIGO interferometers.

Progress in this effort is summarized in Figures 5 - 7. Figure 5 displays the spectral sensitivity of the $2 \mathrm{~km}$ interferometer in strain as a function of the gravitational wave frequency at various times in the commissioning between November 2000 and late June 2002. This interferometer has operated in the power-recycled mode with recycling gains in the range of $15-25$. A common mode servo has been implemented in which the average arm length is used to stabilize the frequency of the laser beam entering the interferometer. Tidal stretching of the arms is sensed and fed to actuators that control the interferometer lock throughout the tidal phases. Lock durations as long as 15 hours have been achieved with this system. The high frequency sensitivity reflects the fact that $5 \mathrm{~W}$ of laser power are entering the interferometer. Sensitivity at low frequencies is limited at this time by technical noise in the sensing and control systems.

Figure 6 is a comparable plot for the second interferometer brought into operation, the Livingston $4 \mathrm{~km}$ instrument. It has reached sensitivity comparable to the Hanford $2 \mathrm{~km}$ interferometer as desired for initiating coincidence science operations between the two observatories. This interferometer is currently being operated with $1.9 \mathrm{~W}$ entering through the recycling mirror and has demonstrated a recycling gain of $\sim 50$. Higher than expected seismic disturbances from local community activities is disturbing the interferometer during daytime hours, resulting in the excitation of seismic isolation system resonances near $2 \mathrm{~Hz}$. For this reason, the best operation has been achieved during the nights, holidays and weekends. Locked periods of the recycled interferometer lasting 4 hours have been achieved during these quieter periods. Tidal feedback control is operational and the arm end mirrors are under alignment control using wavefront sensing. 


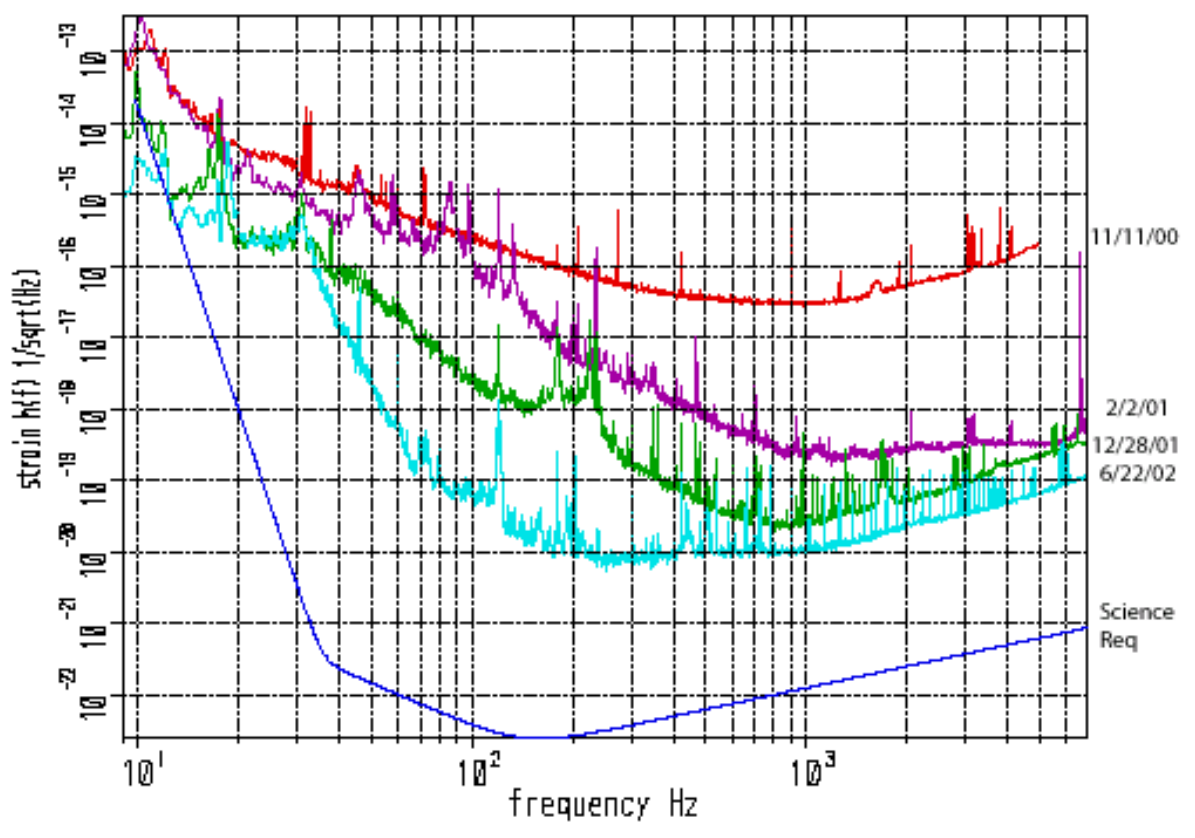

Figure 5 Spectral strain sensitivity of the Hanford $2 \mathrm{~km}$ interferometer at various dates as the commissioning progresses, compared to the Science Requirement for a LIGO 4 km interferometer.

Reduction of the Livingston regional seismic disturbances is being addressed by several mitigation techniques. A global measure, feedforward control of microseismic disturbances, has been introduced first at Livingston in order to reduce the dynamic range required from the controls. A geophone-piezoelectric actuator preisolator system has been employed on the arm cavity end mirrors to significantly reduce the disturbances in the $0.3-5 \mathrm{~Hz}$ range. This has enabled significantly greater locked duty cycle. The system is now installed, as well, on the arm cavity input mirrors and is undergoing commissioning. Finally, a broader engineering solution to the problem is underway to develop two active 6 degree-offreedom preisolation systems, one employing a hydraulic actuator ${ }^{8}$ and a magnetically actuated alternate ${ }^{9}$. One of these systems will be installed early in 2003, following the second science run discussed below, and it is expected that this measure will facilitate locked operation at all times.

Finally, Figure 7 displays the achieved strain sensitivity on the last of the three interferometers, the Hanford $4 \mathrm{~km}$ system. Its installation into the vacuum system was completed during the summer of 2001 and it was brought into full operation in December of 2001. The progress in lowering the strain noise floor is shown. Commissioning of this interferometer has concentrated on a significant new feature, fully digital suspension controls, introduced first in this third system. This powerful capability provides great flexibility and agility in tuning the local servo controls to achieve reliability and low noise. In particular, it permits control of couplings between degrees of freedom in a frequency dependent manner. Once fully commissioned in this system, it will be implemented in the first two interferometers. The displayed slow progress in low frequency sensitivity reflects the attention to commissioning the digital control system. The Hanford $4 \mathrm{~km}$ interferometer has been operated for several hour periods in the recycled configuration with a recycling gain typically $40-50$, with tidal feedback operating and with $1 \mathrm{~W}$ entering the interferometer. 


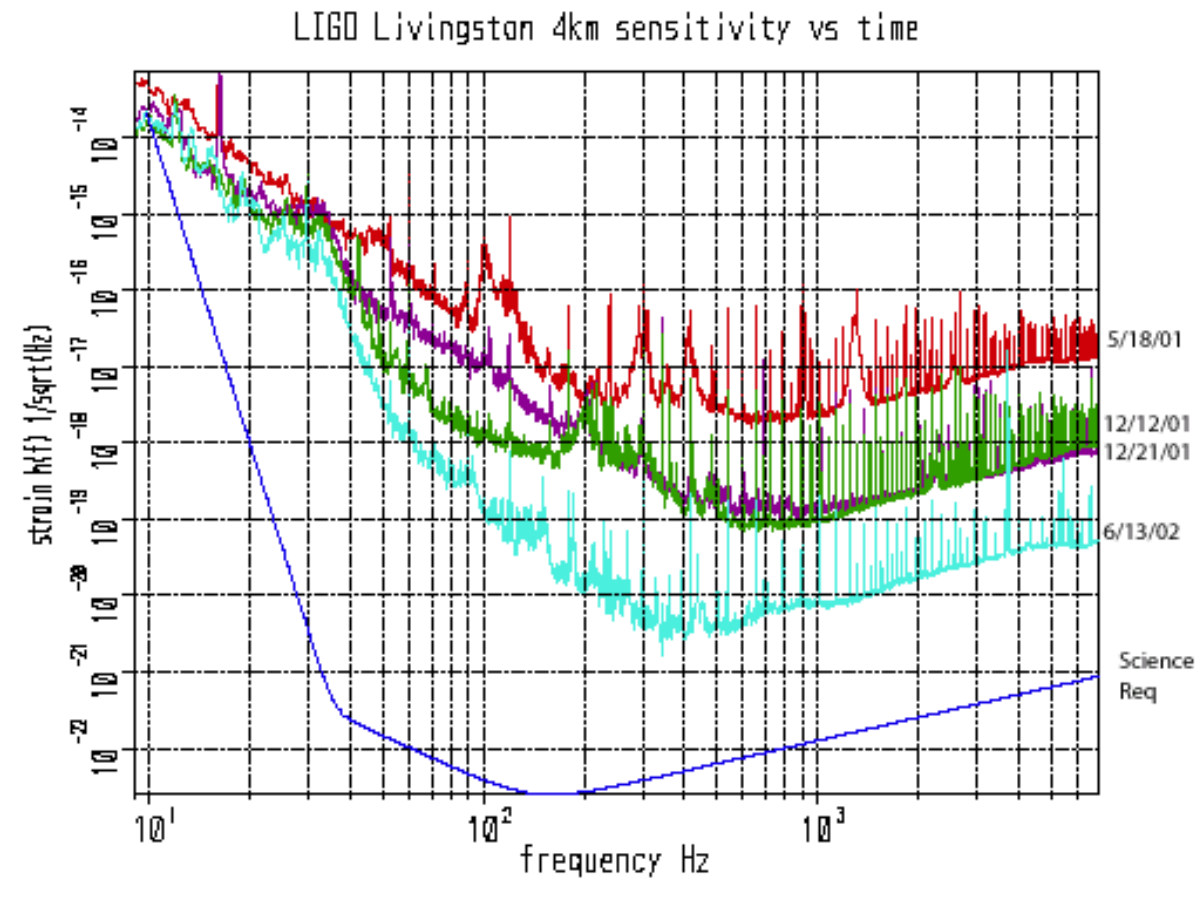

Figure 6 Spectral strain sensitivity of the Livingston $4 \mathrm{~km}$ interferometer at various dates as the commissioning progresses, compared to the Science Requirement for a LIGO 4 km interferometer.

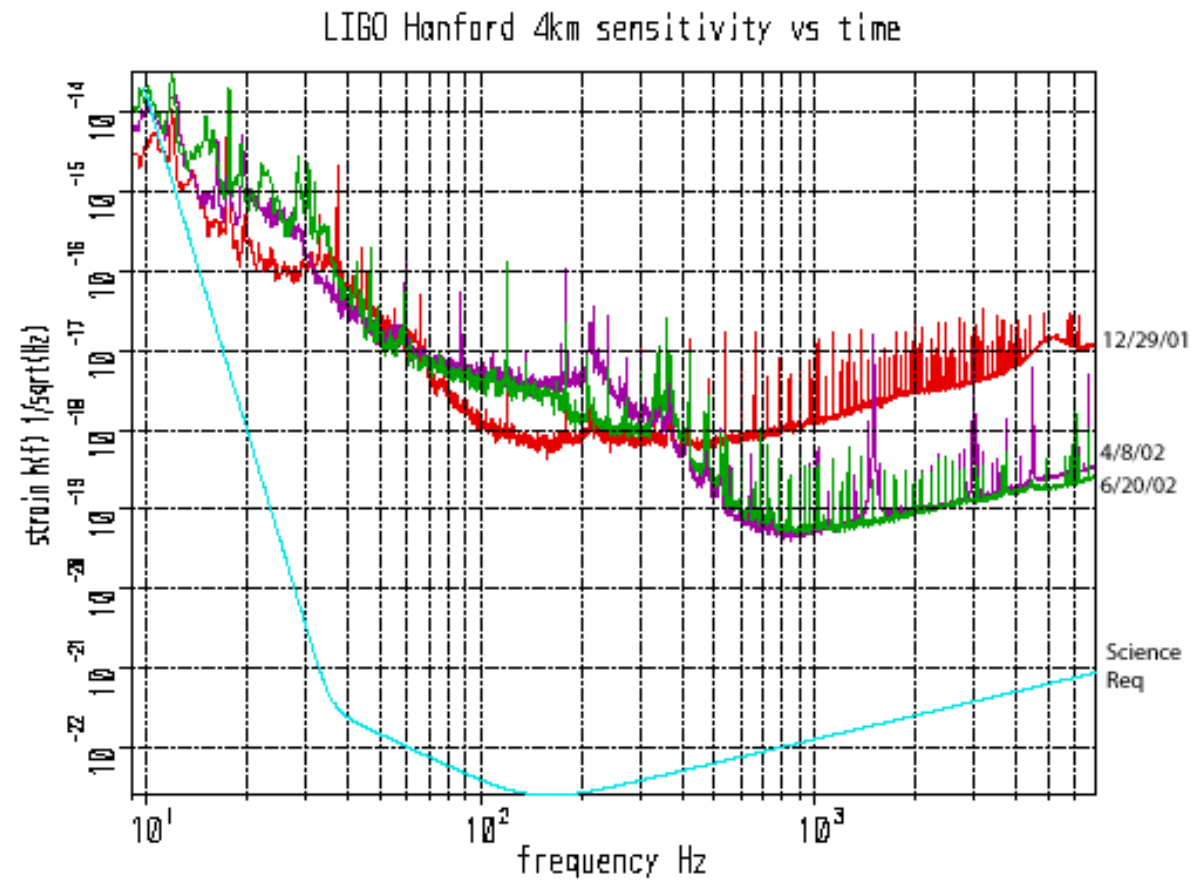

Figure 7 Spectral strain sensitivity of the Hanford $2 \mathrm{~km}$ interferometer at various dates as the commissioning progresses, compared to the Science Requirement for a LIGO 4 km interferometer. 
Through 2002 and 2003, interleaved with the first 3 science runs of LIGO, this commissioning program will continue with the goal of reaching the LIGO Science Requirements ${ }^{5}$ for detector sensitivity and availability. The environmental and detector characterization data collected with science running will contribute insights for this commissioning apart from the value to understanding the scientific import of the data.

\section{A LONG-BASELINE COINCIDENCE ENGINEERING RUN}

A major milestone in the LIGO program was reached on 28 December 2001 when the first simultaneous and extended operation of all 3 interferometers began with a LIGO engineering run dubbed E7 for its place in the sequence of engineering runs. E7 was intended to be a full exercise of the 3 interferometers running around the clock, with all controls and data acquisition systems operating as if the observatories were being run for science purposes. E7 also included a full exercise of the off-line LIGO Data Analysis System (LDAS) from raw data frames through the entire analysis pipeline. Detector sensitivity was very modest and the sensitivities in December 2001 are displayed in Figures 5, 6 and 7. The emphasis placed during preparations immediately prior to this run was on reliability in maintaining servo control lock in order to maximize the time operating in double and triple coincidence mode. High value was placed on acquiring significant periods of data in coincidence in order to fully exercise the data analysis system and to study correlations between interferometer data streams.

In addition to operating the three LIGO interferometers in coincidence, E7 addressed a major goal of the LIGO Scientific Collaboration (LSC) to commission a wider global network of detectors. For this reason, the GEO 600 detector $^{10}$, located near Hannover, Germany was also operated during this period. In addition, the resonant mass detector, ALLEGRO ${ }^{11}$, located at Louisiana State University near the Livingston site, also operated during the E7 period. This detector has been installed on a rotating platform permitting it to be operated at variable orientations with respect to the LIGO Livingston orientation. This permits the pair of detectors (Allegro and LIGO Livingston) to search for remnant Big Bang "stochastic" gravitational waves ${ }^{12}$ in several orientations exploiting the resulting modulation of the signal to strengthen the identification of these waves ${ }^{13}$.

Table 2 summarizes the operating statistics of the three LIGO interferometers and the GEO 600 detector during E7.

Table 2 Engineering Run E7 Run Summary Statistics. The Hanford 2 km (H2), Livingston 4 km (L1) and Hanford 4 km (H1) interferometers were operated simultaneously for various periods. In addition, the GEO 600 and Allegro detectors operated during E7.

28 Dec 2001 - 14 Jan 2002 (402 hr)
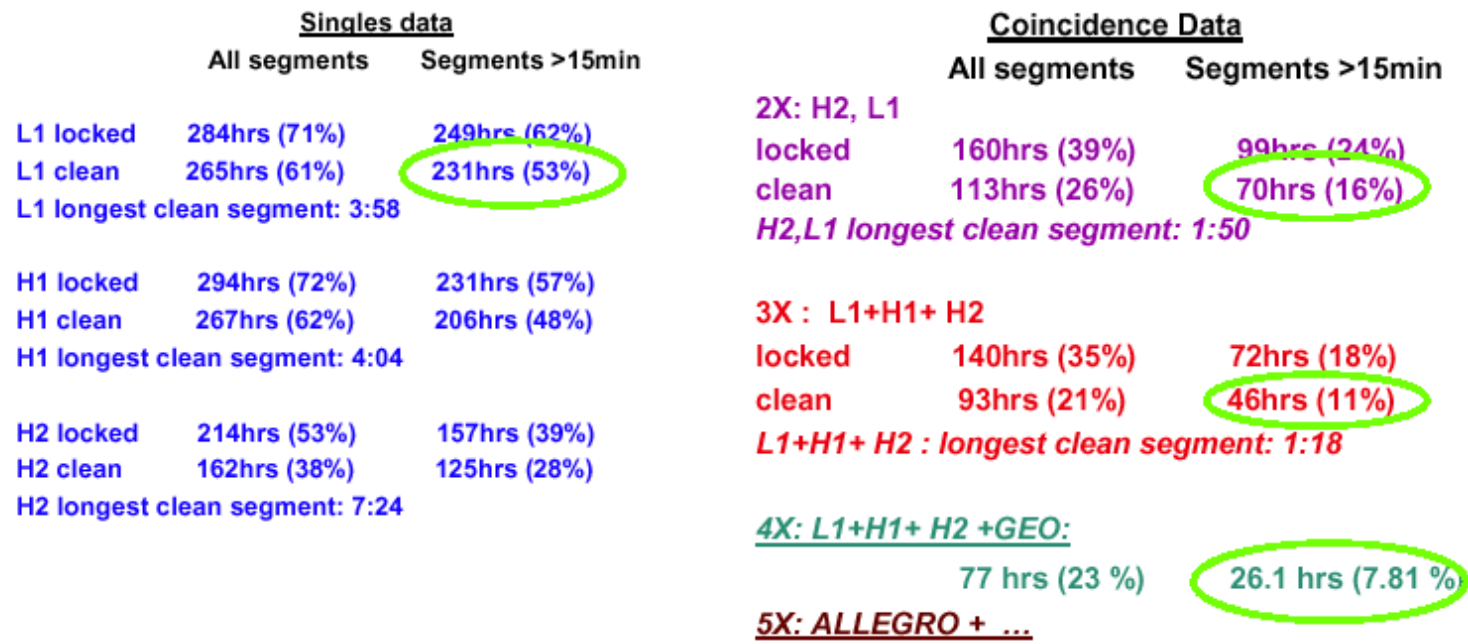
Of the 402 hours of the E7 run, the table displays the number of hours in which individual interferometers, as well as pairs and triples of interferometers, were operated in coincidence. The table lists these periods for all locked segments and for those in which the locked segments were at least 15 minutes long. The latter criterion selects the most useful periods for scientific data as these segments represent operation when the locked interferometer state has settled from the transient behavior observed immediately after lock acquisition. All 3 interferometers operated for 46 hours in coincidence, with a single coincidence segment as long as 1:18 hours. The coincidence of these 3 interferometers with GEO was accomplished for 26.1 hours, representing the first truly long-baseline global operation of this generation of interferometers. The data collected from these periods, and from the operation with Allegro, is currently being analyzed for detector performance and correlation studies and for exercise of the physics analysis methods.

The LDAS system was fully exercised during this run. More than 500,000 files comprising 40 TB were archived in the data archive at Caltech, representing approximately $10 \%$ of the data from an entire year's running. The system demonstrated high reliability and is currently serving the LSC analysis teams.

\section{LIGO DATA ANALYSIS}

LIGO data analysis is currently organized through several working groups of the LSC. The Detector Characterization Working Group analyzes LIGO data with emphasis on understanding the environmental conditions, detector properties, sensitivity and noise performance. Astrophysical signals cannot be verified or interpreted without a full understanding of these detector properties. The astrophysics analysis is organized into four "upper limits" working groups reflecting the current expectation that early science data from LIGO will serve only to set upper limits on the rates and strengths of the several classes of anticipated sources.

The Burst Source upper limit group searches for transient signals from unmodeled sources or sources whose properties are not as well defined as the other three classes. The analysis currently is concentrating on four main classes of filters for such signals. These include searches for events signaled by gamma ray bursts observed by others ${ }^{14}$, and filters based upon statistical studies of time-frequency clustering ${ }^{15}$, the statistics of excess power ${ }^{16}$ in the signal, and a class of filters in the time domain ${ }^{17}$ such as slope detection. The analysis work of this group on the E7 data is complicated by the high level of instrumental transients in this early data and the early experience with tuning this type of analysis. The work continues in preparation for the analysis of data from the science running.

The Periodic Source upper limit group is searching for three classes of sources, employing four techniques. The canonical source produces a continuous wave signal. Such a source could be a non-axisymmetric pulsar. The working group is preparing to search for known pulsars using a coherence search in the frequency domain and a narrow bandwidth heterodyne technique. Unbiased all sky searches are to be carried out using power spectra sums even before Doppler corrections are made. Wide area searches are planned using a hierarchical technique employing Hough transforms ${ }^{18}$.

The Inspiral upper limit group is preparing to search using templates that model the inspiral "chirp" signal in a conventional optimal Wiener filter method ${ }^{19}$ and a newer fast chirp transform technique ${ }^{20}$.

Finally, the Stochastic upper limit group is searching for remnant primordial gravitational waves by cross-correlating the outputs of the various pairs of interferometers and the ALLEGRO data is to be included as well. The E7 data is not able to access physically meaningful sensitivities but the dataset is a test bed for several correlation studies.

\section{INITIATING SCIENTIFIC RUNNING OF LIGO}

The efficacy of a scientific observation period is dependent upon the interferometer sensitivities, the duration of the overlapping periods of data collection, and the total time observing. These depend upon the interferometer noise performance and the period of system "availability" or continuous reliable operation. Postponing scientific operation until sensitivity and availability meet the Science Requirements would delay interesting scientific research and diminish 
the opportunity to perfect data analysis techniques and algorithms. It would also reduce the insights into interferometer performance that derive best from full and careful scientific analysis of the data.

For these reasons, the LIGO Laboratory has planned the initiation of scientific operations to be an interleaved process in which progress in detector commissioning is interleaved with scientific running of the system, with each kind of operation informing and strengthening the other. After a successful series of engineering runs that progressively approached full scientific operations, the E7 run was a "dress rehearsal" for a scientific run. E7 exercised the hardware and data analysis systems and provided an experience base for the LIGO research community.

The initiation of scientific running of LIGO has been planned with two "upper limits" science runs, S1 and S2, interleaved with periods of interferometer commissioning. S1 is planned to occur in summer 2002. The sensitivity goal is a two-site coincidence with three interferometers running and the achieved scientific reach (volume of the universe searched $\mathrm{x}$ observation time in coincidence) is planned to be an order of magnitude better than achieved in the E7 run. At least one interferometer at each site will be operated in the power-recycled configuration.

Following a several month period for data analysis leading to publishable results, and to allow additional interferometer commissioning to be accomplished, the second science run, S2, will take place near the end of 2002. The scientific reach for this run is planned to be an additional order of magnitude improvement in the volume of the universe searched multiplied by the coincidence observation time. These two runs will complete the upper limit running and the orientation for the LIGO running experience. They should lead to a broad set of new publishable limits, well beyond what has been previously published.

An extended commissioning and analysis period will follow S2. During the second half of 2003, LIGO will initiate an extended scientific run, S3, lasting several months. The S3 run will mark the beginning of true search running, representing a step beyond setting upper limits on selected gravitational wave searches. S3 is intended to accomplish a real search for gravitational waves with astrophysical significance. The scientific reach goal for S3 will similarly be an additional order of magnitude beyond S2 in volume of the universe searched multiplied by the duration of the search.

The progression to S3 marks the beginning of very long duration scientific running. The Science Requirements goals will require running most of each year. By the end of 2006, LIGO plans to carry out an observation run that is an integrated year at design sensitivity. That goal represents the mature exploitation of the initial LIGO detectors and the final phase of the initial LIGO Science Run.

\section{FULLY EXPLOITING LIGO}

As indicated in Figure 3, the LIGO facility is intended to support future upgrades and detectors that practically exploit the terrestrial limits on gravitational wave measurement. The ultimate limit is the classical gravity gradients produced by the variability of nearby mass distributions. By the completion of the initial LIGO Science Run, a new generation of interferometer will be developed and can be ready for installation to advance in the exploitation of the LIGO platform. That upgrade is discussed elsewhere in this meeting ${ }^{4}$.

\section{ACKNOWLEDGMENTS}

This paper briefly describes the current status of the efforts by a remarkable group of people, the staff and collaborators in the LIGO effort. There is a great deal of admirable work that cannot be described in this short conference paper. The US National Science Foundation, under awards PHY-9210038 and PHY-0107417, has supported the LIGO effort.

\section{REFERENCES}

\footnotetext{
${ }^{1}$ R. A. Hulse, J. H. Taylor, Astrophys. J. 195, L51 (1975); T. Damour, J. H. Taylor, "On the Orbital Period Change of the Binary Pulsar PSR 1913+16," Astrophys. J. 366, 501 (1991); J. M. Weisberg, J. H. Taylor, "Observations of PostNewtonian Timing Effects in the Binary Pulsar PSR 1913+16," Phys. Rev. Lett. 52, 1348 (1984).

${ }^{2}$ K. S. Thorne, in 300 Years of Gravitation, S. Hawking, W. Israel, eds., Cambridge U. P., Cambridge, England (1987), chap. 9.
} 
${ }^{3}$ A.Abramovici, W.E.Althouse, R.W.P.Drever, Y.Gursel, S.Kawamura, F.J.Raab, D.Shoemaker, L.Sievers, R.E.Spero, K.S.Thorne, R.E.Vogt, R.Weiss, S.E.Whitcomb, and M.E.Zucker, "LIGO -the laser interferometer gravitational-wave observatory," Science 256325 -333 (1992).

${ }^{4}$ P. Fritschel, "Second Generation Gravitational Wave Detectors", Proceedings of SPIE Volume 4856, Gravitational Wave Detection, Waikoloa, HI, 2002, 4856-39.

${ }^{5}$ A. Lazzarini, R. Weiss, "LIGO Science Requirements Document (SRD)," LIGO-E950018-02-E, internal report LIGO Laboratory, California Institute of Technology, MC 18-34, Pasadena, CA 91125, USA and available at http://www.ligo.caltech.edu/docs/E/E950018-02.pdf

${ }^{6}$ P. Brady and T. Creighton, "Searching for periodic sources with LIGO. II. Hierarchical searches", Phys. Rev. D, 61, 2000, 082001; D. I. Jones, "Gravitational waves from rotating neutron stars", Class.Quant.Grav. 19 (2002) 1255-1266, gr-qc/0111007

${ }^{7}$ R. Weiss, "Electromagnetically coupled broadband gravitational antennae, ”MIT Res.Lab.Electron.Q.Prog.Rep.105 $54-76$ (1972).

${ }^{8}$ R. Abbott, R. Adhikari, G. Allen, S. Cowley, E Daw, D. DeBra, J. Giaime, G. Hammond, M. Hammond, C. Hardham, J. How, W. Hua, W. Johnson, B. Lantz, K. Mason, R. Mittleman, J.Nichol, S. Richman, J. Rollins, D. Shoemaker, G. Stapfer, and R Stebbins, "Seismic Isolation for Advanced LIGO", Class. Quant. Gravity 19 (2002) 1591; "Quiet Hydraulic Actuators for Initial LIGO [HEPI]", internal LIGO report at http://www.ligo.caltech.edu/docs/T/T020047$00 . \mathrm{pdf}$

9 "Pre-isolator with Electromagnetic Actuator [MEPI]", internal LIGO report at http://www.ligo.caltech.edu/docs/T/T020041-01.pdf

${ }^{10}$ K.Danzmann, "GEO 600 - A 600-m Laser Interferometric Gravitational Wave Antenna," in First Edoardo Amaldi Conference on gravitational wave experiments E.Coccia, G.Pizella and F.Ronga,eds.(World Scientific, Singapore, 1995), p.100-111.

${ }^{11}$ http://gravity.phys.lsu.edu/

12 J. D. Romano, "A data analysis technique for detecting a stochastic background of gravitational radiation", Proceedings of SPIE Volume 4856, Gravitational Wave Detection, Waikoloa, HI, 2002, 4856-29.

${ }^{13}$ L. S. Finn and A. Lazzarini, "Modulating the Experimental Signature of a Stochastic Gravitational Wave Background", Phys. Rev. D, 64, 15 October 2001, 082002, available at http://www.ligo.caltech.edu/docs/P/P000012A.pdf

${ }^{14}$ L. S. Finn, S. Mohanty, and J. Romano, "Detecting an association between gamma ray and gravitational wave bursts" 30 March 1999, gr-qc/ 9903101; S. Marka et al. at http://www.ligo.caltech.edu/docs/G/G000313-00.pdf

${ }^{15} \mathrm{~J}$. Sylvestre at http://www.ligo.caltech.edu/docs/G/G020028-00.pdf

${ }^{16}$ Warren G. Anderson, Patrick R. Brady, Jolien D. E. Creighton, Eanna E. Flanagan, "A power filter for the detection of burst sources of gravitational radiation in interferometric detectors", Int.J.Mod.Phys. D9 (2000) 303-307 in grqc/0001044

${ }^{17}$ Thierry Pradier, Nicolas Arnaud, Marie-Anne Bizouard, Fabien Cavalier, Michel Davier, Patrice Hello, “An efficient filter for detecting gravitational wave bursts in interferometric detectors", Phys.Rev. D63 (2001) 042002 also in grqc/0010037

${ }^{18}$ B. Schutz and M. Papa, "End-to-end algorithm for hierarchical area searches for long-duration GW sources for GEO 600", proceedings of Jan 1999 Moriond meeting "Gravitational Waves and Experimental Gravity", also in grqc/9905018; P. R. Williams and B. Schutz, "An efficient Matched Filtering Algorithm for the Detection of Continuous Gravitational Wave Signals", proceedings of the 3rd Amaldi conference on gravitational waves, also in gr-qc/9912029

${ }^{19}$ B. Owen, "Search templates for gravitational waves from inspiraling binaries: Choice of template spacing", Phys Rev D, 53, No. 12 (1996) 6749-6761; B.S.. Sathyaprakash, "Filtering post-Newtonian gravitational waves from coalescing binaries “, Phys Rev D, 50, No. 12 (1994) R7111-R7115.

${ }^{20}$ F. A. Jenet and T. A. Prince, "Detection of variable frequency signals using a fast chirp transform", Phys.Rev. D62 (2000), 122001 also in gr-qc/0012029; 